IJMMS 31:1 (2002) 23-29

PII. S0161171202110398

http://ijmms.hindawi.com

(c) Hindawi Publishing Corp.

\title{
ASYMPTOTIC HÖLDER ABSOLUTE VALUES
}

\section{E. MUÑOZ GARCIA}

Received 12 October 2001

\begin{abstract}
We prove that asymptotic Hölder absolute values are Hölder equivalent to classical absolute values. As a corollary we obtain a generalization of Ostrowski's theorem and a classical theorem by E. Artin. The theorem presented implies a new, more flexible, definition of classical absolute value.
\end{abstract}

2000 Mathematics Subject Classification: 12J20, 12J10, 16W80, 13J99.

1. Introduction. Asymptotic Hölder absolute values generalize the notions of classical absolute value and of Hölder absolute value. A Hölder absolute value (HAV) satisfies an approximate triangle inequality and multiplicative property. More precisely, let $C_{1} \geq 1$ and $C_{2} \geq 1$. A $\left(C_{1}, C_{2}\right)$-Hölder absolute value on a ring $R$ is a mapping $\|\cdot\|: R \rightarrow \mathbb{R}_{+}$satisfying:

(HAV1) for $x \in R,\|x\|=0 \Leftrightarrow x=0$;

(HAV2) for $x, y \in R,\|x+y\| \leq C_{2}(\|x\|+\|y\|)$;

(HAV3) for $x, y \in R, C_{1}^{-1}\|x\|\|y\| \leq\|x y\| \leq C_{1}\|x\|\|y\|$.

It is known that HAV on a ring are Hölder equivalent to a classical ones. More precisely, we have the following theorem (see [2]).

THEOREM 1.1 (Hölder rigidity). Let $\|\cdot\|: R \rightarrow \mathbb{R}_{+}$be a $\left(C_{1}, C_{2}\right)$-Hölder absolute value on a commutative ring $R$ with unit element. There exists an absolute value on $R,|\cdot|$ : $R \rightarrow \mathbb{R}_{+}$, which is $\left(C_{1}^{\alpha}, \alpha\right)$-Hölder equivalent to $\|\cdot\|$ with $\alpha=\log _{2}\left(2 C_{2}\right)$, that is, for $x \in R$,

$$
C_{1}^{-\alpha}|x|^{\alpha} \leq\|x\| \leq C_{1}^{\alpha}|x|^{\alpha}
$$

Moreover, $|\cdot|$ can be defined by

$$
|x|=\lim _{n \rightarrow+\infty}\left\|x^{n}\right\|^{1 / n a} .
$$

For a ring $R$ with unity, a real constant $C_{2} \geq 1$, and a function $C_{1}(\cdot, \cdot)$ defined on ] $1,+\infty\left[\times \mathbb{N}\right.$ taking values in $\left[1,+\infty\left[\right.\right.$, we define a $\left(C_{1}, C_{2}\right)$-asymptotic Hölder absolute value (AHAV) on $R$,

$$
|\cdot|: R \rightarrow \mathbb{R}_{+},
$$

satisfying the three following axioms:

(AHAV1) $|x|=0$ if and only if $x=0$;

(AHAV2) for $x, y \in R,|x+y| \leq C_{2}(|x|+|y|)$; 
(AHAV3) for $\gamma>1$ and $n \geq 2$ there is a constant $C_{1}(\gamma, n)>1$ such that for $x_{1}, \ldots$, $x_{n} \in R$,

$$
\begin{aligned}
& C_{1}(\gamma, n)^{-1}\left|x_{1}\right|^{\gamma^{-1}} \cdots\left|x_{n}\right|^{\gamma^{-1}} \leq\left|x_{1} \cdots x_{n}\right| \leq C_{1}(\gamma, n)\left|x_{1}\right|^{\gamma} \cdots\left|x_{n}\right|^{\gamma}, \\
& \quad \text { and } L=\bar{\varlimsup}_{n \rightarrow \infty}(1 / n) \log C_{1}(\gamma, n)<+\infty .
\end{aligned}
$$

We prove the following theorem.

THEOREM 1.2. Let $R$ be a commutative ring with unity. Let $C_{2} \geq 1$ be a real constant, $\alpha=1 / \log _{2}\left(2 C_{2}\right)$, and $\|\cdot\| a\left(C_{1}, C_{2}\right)$-AHAV on $R$. We have the following dichotomy:

(i) if

$$
\varlimsup_{n \rightarrow \infty} \frac{1}{n} \log C_{1}(\gamma, n)=0,
$$

then $\|\cdot\|^{\alpha}$ is a classical absolute value on $R$;

(ii) if

$$
0<L=\varlimsup_{n \rightarrow \infty} \frac{1}{n} \log C_{1}(\gamma, n)<+\infty,
$$

then $\|\cdot\|^{\alpha}$ is a Hölder absolute value on $R$, more precisely, it is $\left(e^{L \alpha}, \alpha\right)$-Hölder equivalent to an absolute value on $R$.

As a result of Theorem 1.2(i), we can define classical absolute values as AHAV with $C_{2}=1$ having a sequence of constants $\left(C_{1}(\gamma, n)\right)_{n}$ growing sub-exponentially, that is,

$$
\varlimsup_{n \rightarrow \infty} \frac{1}{n} \log C_{1}(\gamma, n)=0 .
$$

This is far more flexible than the classical definition.

Note that, in general, Hölder equivalence is a metric property which is stronger than the usual topological equivalence, for example, $\{0\} \cup\{1 / n ; n \geq 1\}$ and $\{0\} \cup\left\{1 / 2^{n}\right.$; $n \geq 1\}$ are homeomorphic, but not Hölder equivalent.

Corollary 1.3. Consider $|\cdot|: R \rightarrow \mathbb{R}^{+}$satisfying

(AV1) $|x|=0$ if and only if $x=0$,

(AV2) for $x, y \in R,|x+y| \leq|x|+|y|$ then,

(AV3) for $x, y \in R,|x y|=|x||y|$ is equivalent to:

(AV3') for $\gamma>1$ and $n \geq 2$ there is a constant $C_{1}(\gamma, n)>1$ such that for $x_{1}, \ldots, x_{n} \in$ $R$,

$$
\begin{aligned}
& C_{1}(\gamma, n)^{-1}\left|x_{1}\right|^{\gamma^{-1}} \cdots\left|x_{n}\right|^{\gamma^{-1}} \leq\left|x_{1} \cdots x_{n}\right| \leq C_{1}(\gamma, n)\left|x_{1}\right|^{\gamma} \cdots\left|x_{n}\right|^{\gamma} \\
& \text { with } \overline{\lim }_{n \rightarrow \infty}(1 / n) \log C_{1}(\gamma, n)=0 .
\end{aligned}
$$

Our theorem gives a generalization for discrete rings of Artin's theorem [1].

COROLLARY 1.4. If $\|\cdot\|$ is a $\left(1, C_{2}\right)$-AHAV over a discrete field $F$, there exists an absolute value $|\cdot|$ and an exponent $\alpha$, such that for all $x$ in $F,\|x\|^{\alpha}=|x|$.

Also, our theorem implies a generalization of Ostrowski's theorem [3] for classical absolute values $\left(C_{1}=C_{2}=\gamma=1\right)$ over $\mathbb{Z}$. 
COROLLARY 1.5. If $\|\cdot\|$ is a $\left(C_{1}, C_{2}\right)$-AHAV over $\mathbb{Z}$ normalized, so that $\|1\|=1$, then $\|\cdot\|$ is $\left(e^{L \alpha}, \alpha\right)$-Hölder equivalent to a $p$-adic absolute value $|\cdot|_{p}$ or to $|\cdot|_{\infty}$ or to the trivial absolute value, with $\alpha=1 / \log _{2}\left(2 C_{2}\right)$.

REMARKS. (1) The constant $C_{1}(\gamma, n)$ in the definition of AHAV can be chosen to satisfy the inequality

$$
C_{1}(\gamma, n) \leq C_{1}\left(\gamma^{1 /\left(\left[\log _{2} n\right]+1\right)}, 2\right)^{n},
$$

where $[a]$ denotes the integer part of $a$.

(2) Let $C_{2} \geq 1$ and let $|\cdot|: R \rightarrow \mathbb{R}_{+}$be a $\left(C_{1}, C_{2}\right)$-AHAV on $R$. If $\varlimsup_{\gamma \rightarrow 1} C_{1}(\gamma, 2)=C_{1}<$ $+\infty$, then $|\cdot|$ is a $\left(C_{1}, C_{2}\right)$-Hölder absolute value.

(3) If $R$ is a ring on which a $\left(C_{1}, C_{2}\right)$-AHAV $|\cdot|$ is defined, then $R$ is a discrete ring for the topology defined by $|\cdot|$.

1.1. Weak subadditive lemma. We prove a generalization of a classical lemma on subadditive sequences (which might be of independent interest).

Definition 1.6. The real sequence $\left(b_{m}\right)_{m \in \mathbb{N}}$ is weakly subadditive if

(i) for $\gamma>1$ and $k \geq 1$, there is a constant $K(\gamma, k)>0$ such that for $m_{1}, \ldots, m_{k} \in \mathbb{N}$,

$$
b_{m_{1}+\cdots+m_{k}} \leq \gamma \sum_{i=1}^{k} b_{m_{i}}+K(\gamma, k) \text {; }
$$

(ii) for $\gamma>1$, we have $K^{*}(\gamma)=\overline{\lim }_{k \rightarrow \infty}(1 / k) K(\gamma, k)<+\infty$.

LEMMA 1.7. If $\left(b_{m}\right)_{m \in \mathbb{N}}$ is weakly subadditive, then

$$
\varliminf_{m \rightarrow \infty} \frac{b_{m}}{m}=\varlimsup_{m \rightarrow \infty} \frac{b_{m}}{m} .
$$

Proof. Fix $n \geq 1$. For any $m \in \mathbb{Z}$, we consider the Euclidean division

$$
m=n q+r, \quad 0 \leq r<n .
$$

Now,

$$
b_{m}=b_{n q+r} \leq \gamma\left(q b_{n}+b_{r}\right)+K(\gamma, q+1) .
$$

Dividing by $m$,

$$
\frac{b_{m}}{m}=\frac{b_{n q+r}}{n q+r} \leq \gamma\left(\frac{q}{n q+r} b_{n}+\frac{b_{r}}{n q+r}\right)+\left(\frac{q+1}{n q+r}\right) \frac{K(\gamma, q+1)}{q+1} .
$$

Taking the upper limit when $m \rightarrow \infty$,

$$
\varlimsup_{m \rightarrow \infty} \frac{b_{m}}{m} \leq \gamma\left(\frac{b_{n}}{n}+0\right)+\frac{1}{n} K^{*}(\gamma) .
$$

That is, for all $q \geq 1$,

$$
\varlimsup_{m \rightarrow \infty} \frac{b_{m}}{m} \leq \gamma \frac{b_{n}}{n}+\frac{1}{n} K^{*}(\gamma) .
$$


Now, taking the lower limit on the right side when $n \rightarrow \infty$,

$$
\varlimsup_{m \rightarrow \infty} \frac{b_{m}}{m} \leq \gamma \varliminf_{n \rightarrow \infty} \frac{b_{n}}{n} .
$$

This holds for all $\gamma>1$, thus making $\gamma \rightarrow 1$,

$$
\varlimsup_{m \rightarrow \infty} \frac{b_{m}}{m} \leq \varliminf_{m \rightarrow \infty} \frac{b_{m}}{m}, \quad \varlimsup_{m \rightarrow \infty} \frac{b_{m}}{m}=\varliminf_{m \rightarrow \infty} \frac{b_{m}}{m} .
$$

\subsection{Proof of Theorem 1.1}

LemmA 1.8. Define $|\|\cdot\||: R \rightarrow \mathbb{R}_{+}$by $|\|x\||=\|x\|^{\alpha}$. Then, $|\|\cdot\||$ is a $\left(C_{1}^{\alpha}, 2\right)$-AHAV on $R$.

Proof. (AHAV1) $|\|x\||=0$ if and only if $\|x\|=0$ if and only if $x=0$.

(AHAV2) $|\|x+y\||=\|x+y\|^{\alpha} \leq\left(2 C_{2}\right)^{\alpha}(\max (\|x\|,\|y\|))^{\alpha} \leq 2\left(\|x\|^{\alpha}+\|y\|^{\alpha}\right)=$ $2(|\|x\||+|\|y\||)$.

(AHAV3) For all $\gamma>1$ and for all $n \geq 2$ there is a constant $C_{1}(\gamma, n)^{\alpha}>1$ such that for all $x_{1}, \ldots, x_{n}$ in $R$,

$$
\begin{aligned}
\left(C_{1}(\gamma, n)\right)^{-\alpha} & \left.|| x_{1}||\right|^{\gamma^{-1}} \cdots\left|\| x_{n}\right|||^{\gamma^{-1}} \\
& \leq||\left|x _ { 1 } \cdots x _ { n } \left\|\left.\left|\leq C_{1}(\gamma, n)^{\alpha}\right||| x_{1}||\right|^{\gamma} \cdots\left|\left\|x_{n}\right\|^{\gamma}\right|^{\gamma} .\right.\right.
\end{aligned}
$$

LEMmA 1.9. Let $x \in R$ and define the real sequence $\left(a_{n}\right)_{n \in \mathbb{N}}$ by $a_{n}=\left|\left\|x^{n}\right\|\right|$. The sequence $\left(a_{n}^{1 / n}\right)$ is converging and

$$
e^{-L}|\|x\|| \leq \lim _{n \rightarrow \infty} a_{n}^{1 / n} \leq e^{L}|\|x\||,
$$

where $L=\varlimsup_{n \rightarrow \infty}(1 / n) \log C_{1}(\gamma, n)<+\infty$.

Proof. Let $b_{m}=\log a_{m}$. The sequence $\left\{b_{m}\right\}$ is weakly subadditive, since for all $\gamma>1$ and for all $k \geq 1$ there is a constant $K(\gamma, k)=\left(C_{1}(\gamma, k)\right)^{\alpha}$, such that

$$
b_{m_{1}+\cdots+m_{k}} \leq \gamma \sum_{i=1}^{k} b_{m_{i}}+\log K(\gamma, k) \text {, }
$$

and for all $\gamma>1$,

$$
\lim _{k \rightarrow+\infty} \frac{1}{k} \log K(\gamma, k)<+\infty
$$

Therefore, by Lemma 1.7,

$$
\varliminf_{m \rightarrow \infty} \frac{b_{m}}{m}=\varlimsup_{m \rightarrow \infty} \frac{b_{m}}{m} .
$$

Thus, to prove the convergence of $\left\{a_{n}^{1 / n}\right\}$, we only have to prove that $\left\{a_{n}^{1 / n}\right\}$ is bounded.

Let $\gamma>1$, for $n \in \mathbb{N}$ there is $C_{1}(\gamma, n)^{\alpha}$ satisfying

$$
C_{1}(\gamma, n)^{-\alpha}|\|x\||^{n / \gamma} \leq\left|\left\|x^{n}\right\|\right| \leq C_{1}(\gamma, n)^{\alpha}|\|x\||^{n \gamma} .
$$


Taking $n$th roots,

$$
C_{1}(\gamma, n)^{-\alpha / n}|\|x\||^{1 / \gamma} \leq a_{n}^{1 / n} \leq C_{1}(\gamma, n)^{\alpha / n}|\|x\||^{\gamma} .
$$

Since $L=\varlimsup_{n \rightarrow \infty}(1 / n) \log C_{1}(\gamma, n)<+\infty$, we obtain

$$
e^{-\alpha L}|\|x\||^{1 / \gamma} \leq \lim _{n \rightarrow \infty} a_{n}^{1 / n} \leq e^{\alpha L}|\|x\||^{\gamma} .
$$

This inequality holds for any $\gamma>1$. Taking the limit when $\gamma \rightarrow 1$,

$$
e^{-\alpha L}|\|x\|| \leq a_{n}^{1 / n} \leq e^{\alpha L}|\|x\|| .
$$

Now we define that $|\cdot|: R \rightarrow \mathbb{R}_{+}$by $|0|=0$ and that $|x|=\lim _{n \rightarrow \infty}\left|\left\|x^{n}\right\|\right|^{1 / n}$ for $x \neq 0$.

LEMmA 1.10. The function $|\cdot|: R \rightarrow \mathbb{R}_{+}$defined as above is an absolute value on $R$. Moreover, if $\overline{\lim }_{n \rightarrow+\infty}(1 / n) \log C_{1}(\gamma, n)=0$, then $|x|=\|x\|^{\alpha}$ for all $x \in R$.

Proof. From Lemma 1.9, if $\overline{\lim }_{n \rightarrow \infty}(1 / n) \log C_{1}(\gamma, n)=0$, we obtain

$$
|\|x\|| \leq|x| \leq|\|x\||
$$

That is, $|x|=\|x\|^{\alpha}$.

It is clear that, $|x|=0$ if and only if $x=0$. Next we check the multiplicative property. For $\gamma>1$ and for $n \geq 2$ there exists $C_{1}(\gamma, 2)^{\alpha}>1$, such that for $n \in \mathbb{N}$ and $x, y$ in $R$,

$$
\begin{aligned}
& C_{1}(\gamma, 2)^{-\alpha}\left|\left\|x^{n}\right\|\right|^{\gamma^{-1}}\left|\left\|y^{n}\right\|\right|^{\gamma^{-1}} \\
& \quad \leq\left|\left\|x^{n}\right\|\right|\left|\left\|y^{n}\right\|\right| \leq C_{1}(\gamma, n)^{\alpha}\left|\left\|x^{n}\right\|\right|^{\gamma}\left|\left\|y^{n}\right\|\right|^{\gamma} .
\end{aligned}
$$

Taking $n$th roots and passing to the limit when $n \rightarrow+\infty$, we obtain

$$
|x|^{\gamma^{-1}}|y|^{\gamma^{-1}} \leq|x y| \leq|x|^{\gamma}|y|^{\gamma} .
$$

Taking the limit when $\gamma \rightarrow 1$, we have the desired multiplicative property.

Finally, we have to check the triangle inequality. This is a corollary of the following general proposition that gives an equivalent, apparently weaker, definition of absolute value.

Proposition 1.11. Let $R$ be a commutative ring with unity. Let $|\cdot|: R \rightarrow \mathbb{R}_{+}$be a function satisfying the following three properties:

(A1) $|x|=0$ if and only if $x=0$;

(A2) (approximate triangle inequality) there exists a real constant $B>0$, such that for all $x, y$ in $R,|x+y| \leq B(|x|+|y|)$;

(A3) for $x, y$ in $R,|x y|=|x||y|$.

Then, $|\cdot|$ is an absolute value on $R$, that is, $|\cdot|$ satisfies the triangle inequality.

LEMMA 1.12. For $x, y \in R$,

$$
|x+y| \leq B(|x|+|y|) \leq 2 B \max (|x|,|y|) .
$$


LEMMA 1.13. Let $|\cdot|^{\prime}: R \rightarrow \mathbb{R}_{+}$, such that for $x, y \in R$,

$$
|x+y|^{\prime} \leq M \max \left(|x|^{\prime},|y|^{\prime}\right),
$$

for some positive constant $M$. Then for $x_{1}, x_{2}, \ldots, x_{n} \in R$,

$$
\left|\sum_{i=1}^{n} x_{i}\right|^{\prime} \leq M^{\left[\log _{2} n\right]+1} \max _{1 \leq i \leq n}\left(\left|x_{i}\right|^{\prime}\right),
$$

where $[a]$ denotes the integer part of $a$.

Proof. Let $m=\left[\log _{2} n\right]+1$ and complete the sequence $\left(x_{i}\right)_{1 \leq i \leq n}$ into $\left(x_{i}\right)_{1 \leq i \leq 2^{m}}$ adjoining 0 elements.

$$
\begin{aligned}
\left|\sum_{i=1}^{2^{m}} x_{i}\right|^{\prime} & \leq M \max \left(\left|\sum_{i=1}^{2^{m-1}} x_{i}\right|^{\prime},\left|\sum_{i=2^{m-1}+1}^{2^{m}} x_{i}\right|^{\prime}\right) \\
& \leq M^{2} \max \left(\left|\sum_{i=1}^{2^{m-2}} x_{i}\right|^{\prime},\left|\sum_{i=2^{m-2}+1}^{2^{m-1}} x_{i}\right|^{\prime},\left|\sum_{i=2^{m-1}+1}^{3 \cdot 2^{m-2}} x_{i}\right|^{\prime},\left|\sum_{i=3 \cdot 2^{m-2}+1}^{2^{m}} x_{i}\right|^{\prime}\right) \\
& \leq \cdots \leq M^{m} \max _{1 \leq i \leq 2^{m}}\left|x_{i}\right|^{\prime} .
\end{aligned}
$$

LEMMA 1.14. Let $\overline{\mathbb{Z}}$ be the image of $\mathbb{Z}$ in $R$. For $n \in \mathbb{N}$,

$$
|\bar{n}| \leq 2 n|1| \text {. }
$$

Proof. We use Lemma 1.13 with $M=2$ and $|\cdot|^{\prime}=|\cdot|$. Take $m=\left[\log _{2} n\right]+1$, $n \leq 2^{m} \leq 2 n$, and $x_{i}=1$ for $1 \leq i \leq n$. We have

$$
|n|=\left|\sum_{i=1}^{n} x_{i}\right| \leq 2^{m}|1| \leq 2 n|1| .
$$

LEMMA 1.15. Let $\overline{\mathbb{Z}}$ be the image of $\mathbb{Z}$ in $R$. For $n \in \mathbb{N}$,

$$
|\bar{n}| \leq n
$$

Proof. Using Lemma 1.14,

$$
\left|\overline{n^{k}}\right|=\left|\bar{n}^{k}\right| \leq 2 n^{k}|1|
$$

and $\left|\bar{n}^{k}\right|^{1 / k} \leq 2^{1 / k} n|1|^{1 / k}$. Taking $k \rightarrow+\infty$, we have $|\bar{n}| \leq n$.

Proof of Proposition 1.11. Let $x, y \in R$ and $n \geq 1$. Let $m=\left[\log _{2} n\right]+1$. Using Lemmas 1.12 and 1.14 , we have

$$
\begin{aligned}
\left|(x+y)^{n}\right| & =\left|\sum_{i=0}^{n}\left(\begin{array}{c}
n \\
i
\end{array}\right) x^{i} y^{n-i}\right| \\
& \leq(B)^{m} \max _{0 \leq i \leq n}\left|\left(\begin{array}{c}
n \\
i
\end{array}\right) x^{i} y^{n-i}\right| .
\end{aligned}
$$


Now using Lemma 1.14,

$$
\begin{aligned}
\left|(x+y)^{n}\right| & \leq(2 B)^{m} \max _{0 \leq i \leq n}\left|\left(\begin{array}{c}
n \\
i
\end{array}\right)\right||x|^{i}|y|^{n-i} \\
& \leq(2 B)^{m} \max _{0 \leq i \leq n}\left(\begin{array}{c}
n \\
i
\end{array}\right)|x|^{i}|y|^{n-i} \\
& \leq(2 B)^{m} \sum_{i=0}^{n}\left(\begin{array}{c}
n \\
i
\end{array}\right)|x|^{i}|y|^{n-i} \\
& \leq(2 B)^{m}(|x|+|y|)^{n} .
\end{aligned}
$$

Finally,

$$
|x+y|=\left|(x+y)^{n}\right|^{1 / n} \leq(2 B)^{(1 / n)\left(\left[\log _{2} n\right]+1\right)}(|x|+|y|),
$$

and passing to the limit $n \rightarrow+\infty$ we get the sharp triangle inequality $|x+y| \leq|x|+|y|$.

\section{PROOF OF THEOREM 1.2.}

CASE 1. Assume $\varlimsup_{n \rightarrow \infty}(1 / n) \log C_{1}(\gamma, n)=0$. By Lemma 1.8, for all $x, y$ in $R$ we have

$$
|x+y|=|\|x+y\|| \leq 2(|\|x\||+|\|y\||) \leq 4 \max (|\|x\||,|\|y\||)=4 \max (|x|,|y|) .
$$

Therefore, by Proposition 1.11, the function $|\cdot|$ satisfies the triangle inequality.

CASE 2. Assume $0<L=\overline{\lim }_{n \rightarrow \infty}(1 / n) \log C_{1}(\gamma, n)<+\infty$. From Lemma 1.9, for any $x$ in $R$,

$$
e^{-\alpha L}|\|x\|| \leq|x| \leq e^{\alpha L}|\|x\||
$$

Therefore,

$$
|x+y| \leq e^{\alpha L}|\|x+y\|| \leq 2 e^{\alpha L}(|\|x\||+|\|y\||) \leq 2 e^{2 \alpha L}(|x|+|y|) .
$$

Thus by Proposition 1.11 , the function $|\cdot|$ satisfies the triangle inequality, it is an absolute value, and $\|\cdot\|^{\alpha}$ is $\left(e^{L \alpha}, \alpha\right)$-equivalent to $|\cdot|$.

\section{REFERENCES}

[1] E. Artin, Theory of Algebraic Numbers, George Striker, Göttingen, 1959.

[2] E. Muñoz Garcia, Hölder absolute values are equivalent to classical ones, Proc. Amer. Math. Soc. 127 (1999), no. 7, 1967-1971.

[3] A. Ostrowski, Über einige Lösungen der Funktionalgleichung $\phi(x) \cdot \phi(y)=\phi(x \cdot y)$, Acta Math. 41 (1914), 271-284 (German).

E . Muñoz Garcia: Department of Mathematics, University of California, Los AngeLES, 405 Hilgard AVEnue, Los ANGeles, CA 90095-1555, USA

E-mail address: munoz@math.uc7a.edu 


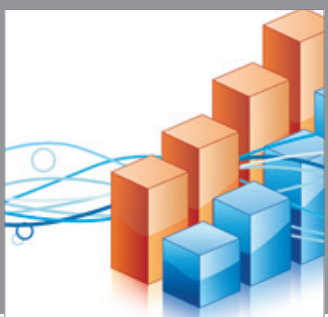

Advances in

Operations Research

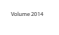

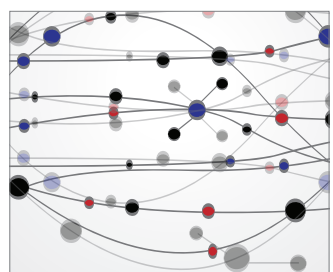

\section{The Scientific} World Journal
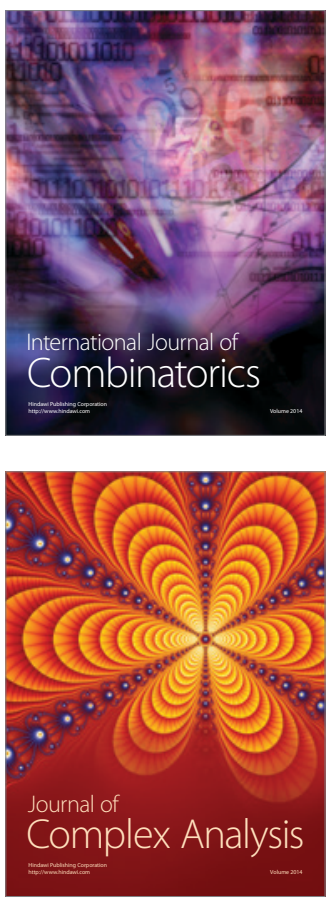

International Journal of

Mathematics and

Mathematical

Sciences
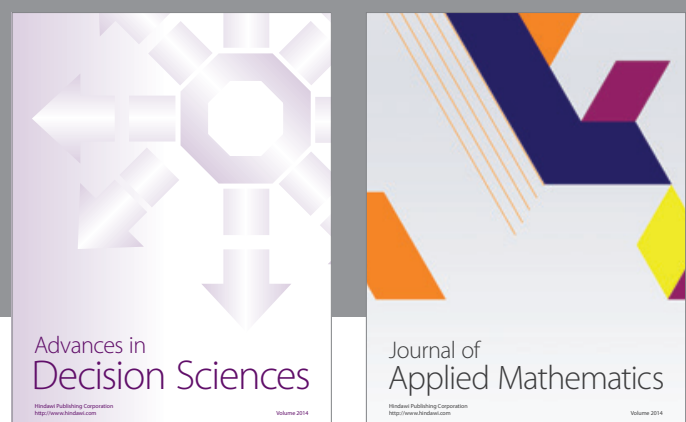

Journal of

Applied Mathematics
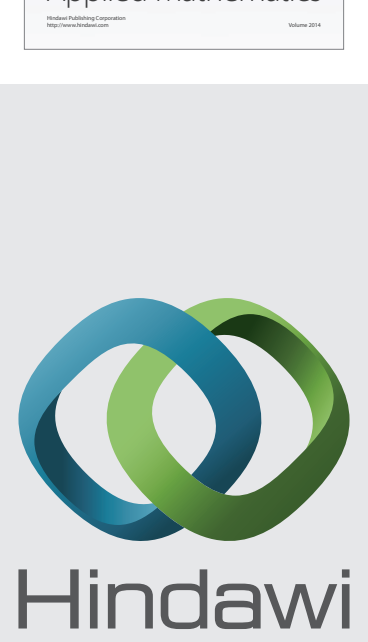

Submit your manuscripts at http://www.hindawi.com
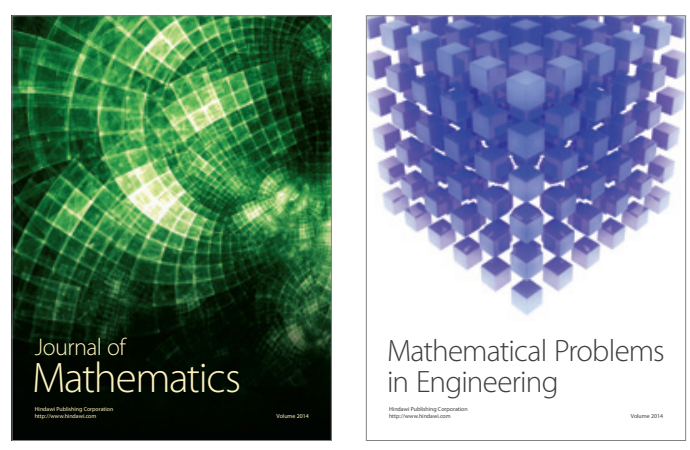

Mathematical Problems in Engineering
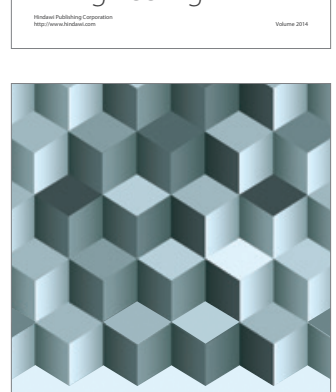

Journal of

Function Spaces
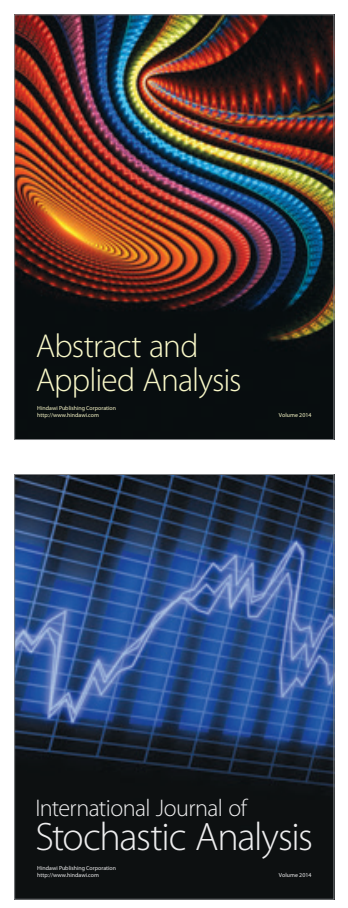

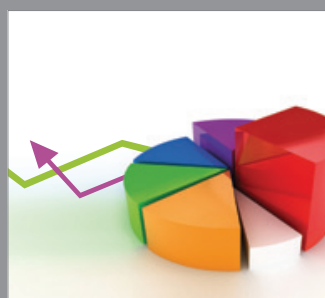

ournal of

Probability and Statistics

Promensencen
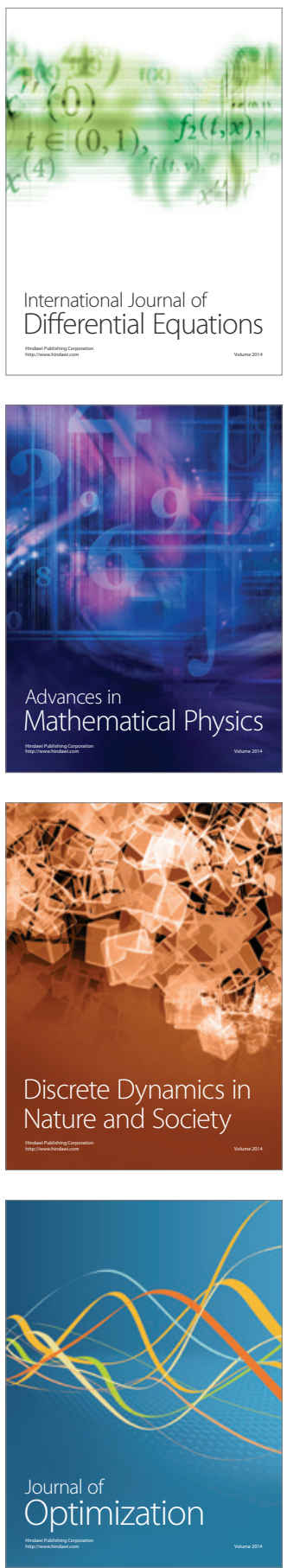